\title{
Impact of previous metronidazole exposure on metronidazole-based second-line quadruple therapy for Helicobacter pylori infection
}

Gil Ho Lee, Kee Myung Lee, Sung Jae Shin, Joon Koo Kang, Choong-Kyun Noh, Jin Hong Kim, and Sun Gyo Lim

Department of Gastroenterology, Ajou University School of Medicine, Suwon, Korea

Received: April 24, 2020

Revised : June 13, 2020

Accepted: July 9, 2020

\section{Correspondence to}

Sun Gyo Lim, M.D.

Department of Gastroenterology,

Ajou University School of

Medicine, 164 World cup-ro,

Yeongtong-gu, Suwon 16499,

Korea

Tel: +82-31-219-6939

Fax: +82-31-219-5999

E-mail: mdlsk75@ajou.ac.kr

https://orcid.org/0000-0003-

2045-5099
Background/Aims: The negative effects on the eradication success of Helicobacter pylori infection after previous exposure to macrolides, including clarithromycin on clarithromycin-based first-line therapy have been demonstrated. However, whether this is true for metronidazole-based second-line quadruple therapy remains unclear. We investigated the relationship between past administration of metronidazole and the failure of metronidazole-based second-line quadruple therapy in patients with $H$. pylori infection.

Methods: Patients over 20 years of age who were diagnosed with $H$. pylori infection between January 1998 and March 2016 were enrolled in this study. The relationship between the clinical parameters and the results of a C13-urea breath test after metronidazole-based second-line quadruple therapy was analyzed in patients for whom clarithromycin-based triple therapy failed to eradicate $H$. pylori.

Results: The $\mathrm{H}$. pylori eradication failure rate was significantly higher in patients with a history of metronidazole use than in patients without a history of metronidazole use $(p=0.011)$. Multivariable analysis showed that the odds ratio of previous metronidazole use for eradication failure was 3.468 (95\% confidence interval, 1.391 to $8.649 ; p=0.008$ ). In the subgroup analysis of patients with a history of metronidazole use, the duration of metronidazole use and interval between its use and eradication therapy did not significantly affect $H$. pylori eradication failure.

Conclusions: Previous exposure to metronidazole was a significant risk factor for treatment failure of metronidazole-based second-line quadruple therapy; therefore, this should be considered when establishing a treatment strategy for patients with $H$. pylori infection.

Keywords: Helicobacter pylori; Drug resistance; Antibiotics; Metronidazole.

\section{INTRODUCTION}

Helicobacter pylori is a gram-negative, microaerophilic bacterium that resides in the stomach and is a major risk factor for gastric neoplastic and peptic ulcer diseas- es $[1,2]$. According to epidemiological and experimental studies, the prevalence of $H$. pylori-related diseases has been decreasing owing to the aggressive eradication therapy for $H$. pylori infection [3]. However, recent studies have demonstrated that the success rates of $H$. pylori 


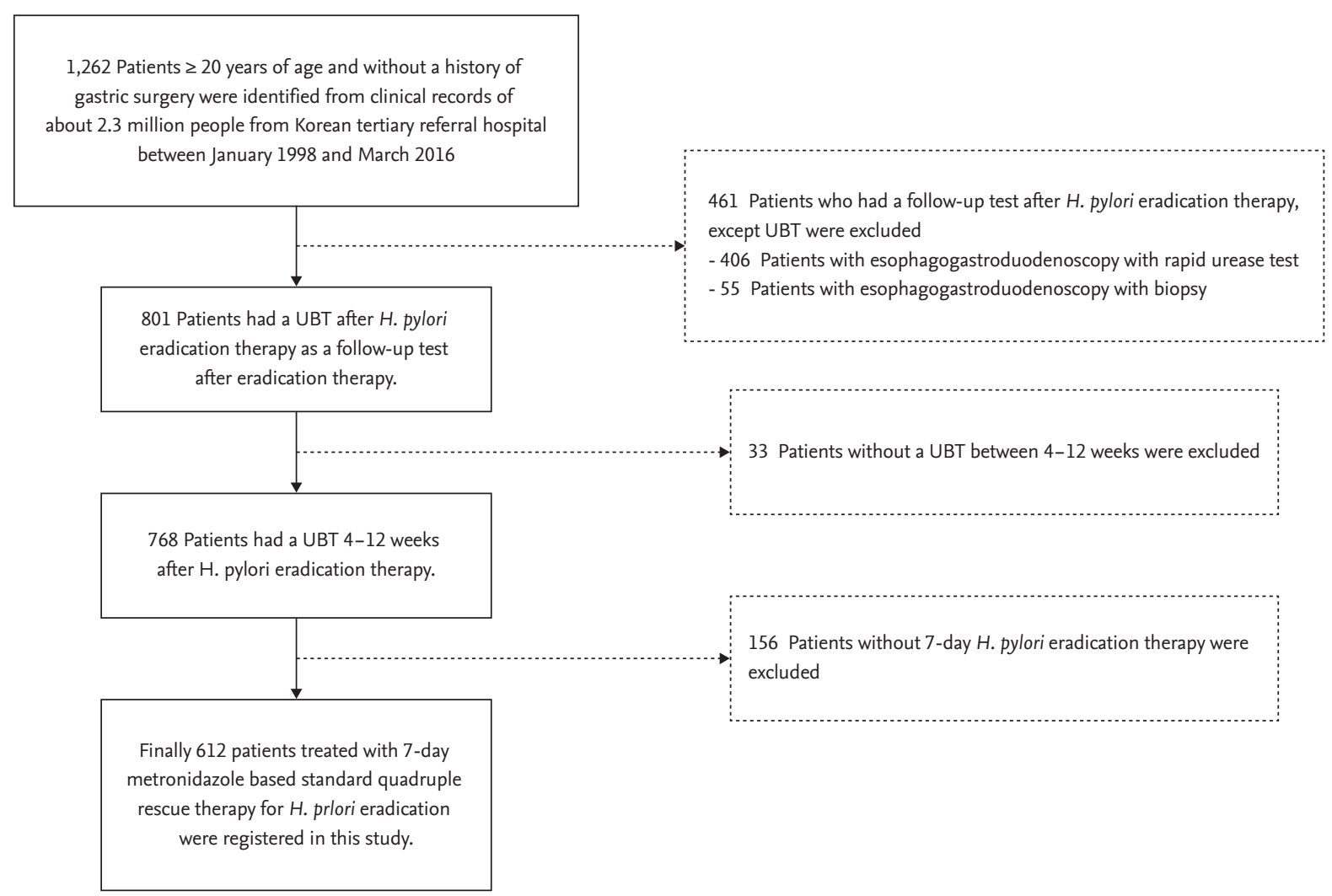

Figure 1. Study protocol sheet. H. pylori, Helicobacter pylori; UBT, urea breath test.

eradication have been gradually decreasing [4-6]. Among the important factors affecting the failure of $\mathrm{H}$. pylori eradication, antibiotic resistance has been consistently reported to be a major cause of treatment failure $[7,8]$.

In Korea, clarithromycin (CAM)-based triple therapy has been recommended as a first-line eradication therapy. A meta-analysis of 104 studies on the Korean standard CAM-based triple therapy and a Korean nationwide multicenter study showed that the success rate of standard triple therapy significantly reduced from 1998 to $2013[6,9,10]$.

Although an antimicrobial susceptibility test is the gold standard for establishing antibiotic resistance, obtaining the equipment for its performance and applying it to clinical practice is usually difficult. Recently, dual priming oligonucleotide-based multiplex polymerase chain reaction was developed to identify CAM resistance and help predict the failure of CAM-based $H$. pylori eradication therapy $[11,12]$. However, a reliable test to identify metronidazole(MET) resistance does not exist. Therefore, clinical information that can predict antibiotic resistance and eradication failure needs to be utilized to provide an individualized treatment strategy for $H$. pylori infection. Previously, we demonstrated that the failure rate of CAMbased first-line eradication therapy was significantly increased in patients with a history of CAM and macrolide use [13]. However, the relationship between past MET use and $H$. pylori eradication failure using a quadruple regimen that includes MET has not been determined.

Therefore, we aimed to investigate the relationship between past use of MET and the failure of MET-based second-line quadruple therapy for $H$. pylori infection. This knowledge would further the ability to provide an individualized treatment strategy for patients with $H$. pylori infection.

\section{METHODS}

\section{Study population}

This retrospective cohort study evaluated whether a previous use of MET affected the failure of second-line 
$H$. pylori eradication therapy. The clinical research database used in this study consists of clinical, prescriptive, laboratory, and administrative data from the records of approximately 2.3 million people observed at a South Korean tertiary teaching hospital (Ajou University Hospital) from January 1998 to March 2016.

The data search and selection process is shown in Fig. 1. All patients over 20 years of age who were diagnosed with $H$. pylori infection between January 1998 and March 2016 were included in the study. $H$. pylori infection was defined as a positive result in a rapid urease test performed by esophagogastroduodenoscopy or in a pathologic analysis of a biopsy specimen. Some patients tested positive in the follow-up urea breath test (UBT; Korea Otsuka Pharmaceutical, Seoul, Korea) 4 to 12 weeks after undergoing first-line 7-day standard triple therapy consisting of $1 \mathrm{~g}$ twice a day amoxicillin (AMX), $500 \mathrm{mg}$ twice a day CAM, and one of the proton pump inhibitors (PPIs; $40 \mathrm{mg}$ pantoprazole, $30 \mathrm{mg}$ lansoprazole, $20 \mathrm{mg}$ esomeprazole, $20 \mathrm{mg}$ rabeprazole; twice daily). Among then, the patients who underwent the 7-day MET-based second-line quadruple therapy (one of the aforementioned PPIs twice daily, $300 \mathrm{mg}$ tripotassium dicitrate bismuthate and $500 \mathrm{mg}$ tetracycline four times daily, and $500 \mathrm{mg}$ MET thrice daily) and underwent follow-up UBT at 4 to 12 weeks were finally included in this study.

The enrolled patients were divided into two groups: patients with a history of MET medication and those without a history of MET medication. The exclusion criteria were age $<20$ years, the absence of a follow-up UBT at 4 to 12 weeks after therapy, or any history of gastric surgery. This study protocol was approved by the Institutional Review Board of Ajou University Hospital (AJIRB-MED-MDB-17-379), and the requirement of informed consent was waived owing to the anonymous nature of the retrospective cohort data.

\section{Data acquisition}

Data regarding the patients' sex, age, underlying disease, history and duration of previous MET use, interval between previous MET use and second-line eradication, presence of gastroduodenal ulcer, and UBT results were obtained. Patients' sex, age, presence of endoscopic gastroduodenal ulcer, and UBT results were obtained by reviewing the medical records, and the interval between previous MET use and second-line eradication and dura- tion of previous MET use were obtained from inpatient and outpatient records. The data of patients with a history of MET use were extracted by searching all MET prescriptions provided in our medical center before administration of the 7-day MET-based second-line quadruple therapy. Underlying disease was identified by the International Classification of Disease 1oth Revision code and the associated test results: diabetes (E10-E11); chronic hepatitis (hepatitis B virus surface antigen + or hepatitis C virus antibody +); liver cirrhosis (K74.6, abdominal ultrasonography); and end-stage renal disease (ESRD, N18.5, if dialysis was present in the medical history).

\section{Main outcomes}

The primary end point was the failure rate of $H$. pylori eradication in both groups. The failure or success of $H$. pylori eradication was determined by the UBT results. The following factors were evaluated as potential risk factors for the failure of second-line quadruple eradication therapy, including MET: history of antibiotic use, age, sex, presence of gastroduodenal ulcer, and underlying disease (diabetes, chronic hepatitis, cirrhosis, and ESRD). We performed additional analyses to determine whether other factors, such as the duration of previous MET use and the interval between previous MET use and second-line eradication, were related to the success rate of eradication treatment.

\section{Statistical analysis}

Descriptive statistics were used to estimate the frequency, mean, and standard deviation of variables. The risk of eradication failure was calculated using a $t$ test for continuous variables and a chi-square test or Fisher's exact test for categorical variables. Determinants with significant associations established by univariate analysis were considered confounding variables and included in a binary multivariable logistic regression analysis. A $p<0.05$ was considered statistically significant in all analyses. Data were analyzed using SPSS version 25 for Windows (IBM Co., Armonk, NY, USA).

\section{RESULTS}

\section{Baseline characteristics}

The demographic and clinical characteristics and the 
Table 1. Baseline characteristics of the patients

\begin{tabular}{|c|c|c|c|c|}
\hline \multirow{2}{*}{ Variable } & \multirow{2}{*}{ Total patients $(\mathrm{n}=612)$} & \multicolumn{2}{|c|}{ Result of $H$. pylori eradication therapy } & \multirow{2}{*}{$p$ value } \\
\hline & & Failure & Success & \\
\hline Age, yr & & & & $0.227^{\mathrm{a}}$ \\
\hline$\leq 60$ & $433(70.8)$ & $33(7 \cdot 6)$ & $400(92.4)$ & \\
\hline$>60$ & $179(29.2)$ & $19(10.6)$ & $160(89.4)$ & \\
\hline Sex & & & & $0.691^{a}$ \\
\hline Male & $337(55 \cdot 1)$ & $30(8.9)$ & $307(91.1)$ & \\
\hline Female & $275(44.9)$ & $22(8.0)$ & $253(92.0)$ & \\
\hline \multicolumn{2}{|l|}{ Diabetes mellitus } & & & $0.565^{b}$ \\
\hline Yes & $40(6.5)$ & $2(5.0)$ & $38(95.0)$ & \\
\hline No & $572(93 \cdot 5)$ & $50(8.7)$ & $522(91.3)$ & \\
\hline \multicolumn{2}{|l|}{ Chronic hepatitis } & & & $1.000^{b}$ \\
\hline Yes & $6(1.0)$ & $\mathrm{O}$ & $6(100.0)$ & \\
\hline No & $606(99.0)$ & $52(8.6)$ & $554(91.4)$ & \\
\hline \multicolumn{2}{|l|}{ Liver cirrhosis } & & & $0.359^{\mathrm{b}}$ \\
\hline Yes & $5(0.8)$ & $1(20.0)$ & $4(80.0)$ & \\
\hline No & $607(99.2)$ & $51(8.4)$ & $556(91.6)$ & \\
\hline \multicolumn{2}{|l|}{ ESRD } & & & $1.000^{b}$ \\
\hline Yes & $6(1.0)$ & o & $6(100.0)$ & \\
\hline No & $606(99.0)$ & $52(8.6)$ & $554(91.4)$ & \\
\hline \multicolumn{2}{|c|}{ Presence of gastroduodenal ulcer } & & & $0.424^{\mathrm{a}}$ \\
\hline Yes & $239(39.1)$ & $23(9.6)$ & $216(90.4)$ & \\
\hline No & $373(60.9)$ & $29(7.8)$ & $344(92.2)$ & \\
\hline \multicolumn{2}{|c|}{ Previous use of metronidazole } & & & $0.011^{b}$ \\
\hline Yes & $31(5 \cdot 1)$ & $7(22.6)$ & $24(77 \cdot 4)$ & \\
\hline No & $581(94.9)$ & $45(7 \cdot 7)$ & $536(92.3)$ & \\
\hline Overall & $612(100)$ & $52(8.5)$ & $560(91.5)$ & \\
\hline
\end{tabular}

Values are presented as number (\%).

H. pylori, Helicobacter pylori; ESRD, end-stage renal disease.

${ }^{a}$ Chi-square test was used for statistical analysis.

${ }^{\mathrm{b}}$ Fisher's exact test was used for statistical analysis.

results of the univariate analysis are presented in Table 1. A total of $6_{12}$ patients underwent second-line eradication therapy with a MET-based quadruple regimen. Of them, 179 (29.2\%) were over 6o years old, and 337 (55.1\%) were male. The rates of the enrolled patients' comorbidities were: diabetes in 40 patients $(6.5 \%)$, chronic hepatitis in six (1.0\%), liver cirrhosis in five (0.8\%), and ESRD in six (1.0\%). A total of 239 patients (39.1\%) had a gastroduodenal ulcer, and 31 (5.1\%) had a history of previous MET use. Overall, the $H$. pylori eradication rate of MET- based second-line quadruple therapy was $91.5 \%$ (560 of 612), while that in patients with a history of MET use was $77.4 \%$ (24 of 31$)$.

Relationship between the failure rate of second-line $H$. pylori eradication therapy and various clinical factors

The failure rate of eradication therapy was not significantly correlated with clinical factors, such as age, sex, underlying disease (diabetes mellitus, chronic hepatitis, 
Table 2. Multivariable analysis of the relationship between clinical factors and the failure of Helicobacter pylori eradication therapy

\begin{tabular}{lcc}
\hline \multirow{2}{*}{ Variable } & \multicolumn{2}{c}{ Binary logistic regression analysis } \\
\cline { 2 - 3 } & OR (95\% CI) & $p$ value \\
\hline Age > 6o yr & $1.019(0.994-1.044)$ & 0.137 \\
Male sex & $1.156(0.639-2.090)$ & 0.631 \\
Diabetes mellitus, yes & $0.514(0.118-2.339)$ & 0.376 \\
Liver cirrhosis, yes & $4.818(0.420-55.212)$ & 0.206 \\
Presence of gastroduodenal ulcer, yes & $1.300(0.720-2.349)$ & 0.384 \\
Previous use of metronidazole, yes & $3.468(1.391-8.649)$ & 0.008 \\
\hline
\end{tabular}

OR, odds ratio; CI, confidence interval.

Table 3. Outcomes of 7-day metronidazole-based 2 nd line quadruple therapy in patients with a history of metronidazole use according to various clinical factors

\begin{tabular}{|c|c|c|c|c|}
\hline \multirow{2}{*}{ Variable } & \multirow{2}{*}{ Total patients $(n=31)$} & \multicolumn{2}{|c|}{ Result of $H$. pylori eradication therapy } & \multirow{2}{*}{$p$ value } \\
\hline & & Failure & Success & \\
\hline Sex & & & & $0.685^{\mathrm{a}}$ \\
\hline Male & 15 & $4(26.7)$ & $11(73 \cdot 3)$ & \\
\hline Female & 16 & $3(18.7)$ & $13(81.3)$ & \\
\hline Reason of MET use & & & & $1.000^{\mathrm{a}}$ \\
\hline Gynecologic infection & 8 & $2(25)$ & $6(75 \cdot 0)$ & \\
\hline Intra-abdominal infection & 20 & $4(20.0)$ & $16(80.0)$ & \\
\hline Others & 3 & $1(33 \cdot 3)$ & $2(66.7)$ & \\
\hline Route of MET administration & & & & $0.412^{\mathrm{a}}$ \\
\hline Intravenous & 14 & $2(14 \cdot 3)$ & $12(85.7)$ & \\
\hline Oral & 17 & $5(29 \cdot 4)$ & $12(70.6)$ & \\
\hline Daily dose of MET & & & & $0.667^{\mathrm{a}}$ \\
\hline$\geq 1,500 \mathrm{mg}$ & 13 & $2(15 \cdot 4)$ & $11(84.6)$ & \\
\hline$<1,500 \mathrm{mg}$ & 18 & $5(27.8)$ & $13(72.2)$ & \\
\hline MET use during operation & & & & $1.000^{\mathrm{a}}$ \\
\hline Yes & 11 & $2(18.2)$ & $9(81.8)$ & \\
\hline No & 20 & $5(25)$ & $15(75.0)$ & \\
\hline
\end{tabular}

Values are presented as number (\%).

H. pylori, Helicobacter pylori; MET, metronidazole.

${ }^{\text {a}}$ Fisher's exact test was used for statistical analysis.

liver cirrhosis, ESRD), or the presence of a gastroduodenal ulcer. The failure rate of eradication therapy was significantly higher in patients with previous MET use than in patients without a history of MET use (22.6\% vs. $7.7 \%, p=0.011$ ). Multivariable binary logistic regression analysis showed that the odds ratio of past MET use for treatment failure was 3.468 (95\% confidence interval,
1.391 to $8.649 ; p=0.008$ ) (Table 2). Among the 31 patients with a history of MET use, clinical factors, such as sex, reason of MET use, route of MET administration, daily dose of MET, and MET use during operation did not significantly affect the treatment outcome of the 7-day MET-based second-line $H$. pylori eradication therapy (Table 3). 
Table 4. Results of Helicobacter pylori eradication therapy in patients with a history of metronidazole use

\begin{tabular}{|c|c|c|c|c|}
\hline \multirow{2}{*}{ Variable } & \multirow{2}{*}{ Overall no. of patients $(n=31)$} & \multicolumn{2}{|c|}{ Result of urea breath test } & \multirow{2}{*}{$p$ value } \\
\hline & & Failures & Success & \\
\hline Duration of MET use & & & & $0.053^{\mathrm{a}}$ \\
\hline$\geq 14$ day & $8(25.8)$ & $4(50.0)$ & $4(50.0)$ & \\
\hline$<14$ day & $23(74.2)$ & $3(13.0)$ & $20(87.0)$ & \\
\hline \multicolumn{2}{|c|}{ Interval between MET use and eradication therapy } & & & $1.000^{\mathrm{a}}$ \\
\hline$>1 \mathrm{yr}$ & $22(71.0)$ & $5(22.7)$ & $17(77 \cdot 3)$ & \\
\hline$<1 \mathrm{yr}$ & $9(29.0)$ & $2(22.2)$ & $7(99.8)$ & \\
\hline
\end{tabular}

Values are presented as number (\%).

MET, metronidazole.

${ }^{a}$ Fisher's exact test was used for statistical analysis.

\section{Relationship between the failure rate of $\mathrm{H}$. pylori eradication therapy and the duration of MET use}

An analysis of the effect of the duration of MET use and the interval between MET use and eradication therapy on eradication failure was performed for 31 patients with a history of MET use. The numbers of patients who had taken MET for $\geq 14$ days or for $<14$ days were eight and 23 , respectively. The failure rates of $H$. pylori eradication were $50 \%(4 / 8)$ and $13 \%(3 / 23)$ for the two groups, respectively $(p=0.053)$ (Table 4$)$. As for the effect of the duration of previous MET use, the failure rate of eradication therapy tended to increase in patients with $\geq 14$ days of MET use than in those of patients with $<14$ days of usage $(p=0.05)$.

\section{DISCUSSION}

This retrospective cohort study showed that previous exposure to MET had a significant impact on the failure of MET-based second-line eradication therapy of $H$. pylori. This study is the first to investigate the relationship between previous MET use and the failure of METbased $H$. pylori eradication therapy. Using binary logistic regression analysis for risk assessment, a previous use of MET was identified to be an independent risk factor for the failure of MET-based second-line quadruple therapy. In patients who had a > 14-day history of MET administration, the failure rate of $H$. pylori eradication was significantly increased. These results agreed with those of a previous study, which revealed that a history of mac- rolide treatment was closely related to the increased failure rate of CAM-based H. pylori eradication therapy [14].

According to the Maastricht V/Florence Consensus, after PPI-CAM-AMX triple therapy failure, bismuth quadruple therapy (BQT) or fluoroquinolone-containing triple or quadruple therapy are recommended as second-line treatments [15]. In areas of high CAM and MET resistance, BQT is the recommended first-line regimen in cases of $\mathrm{H}$. pylori infection.

If 7-day CAM-based standard triple therapy fails in patients with a history of MET use, the following strategies are available. First, 14-day CAM-based standard triple therapy could be recommended based on the results of the meta-analyses [16-18]. Second, prolongation of MET-based eradication therapy could be considered. A 10- or 14-day MET-based BQT has been reported to improve the success rate of $H$. pylori eradication [19-22]. However, most of these studies were not performed on the basis of antibiotic susceptibility testing. Therefore, on an individual basis, it is questionable whether the outcomes of 10- or 14-day MET-based second-line quadruple therapy in patients with a history of MET use would be improved, as was observed in patients without a history of MET use. Upon further analysis, among patients who had received 10- or 14-day MET-based BQT in our medical center and whose data were not included in this study, the success rate of $H$. pylori eradication was lower in patients with a history of MET than that of those who had no such history (81.3\% vs $95.2 \%, p=0.54$ ). This result showed that prolongation of MET-based second-line quadruple therapy might not improve the 
success rate of $H$. pylori eradication therapy in patients who had previously taken MET.

MET is activated via reduction in bacteria to produce nitro-anion radicals and imidazole intermediates, which damage bacterial DNA, thus exhibiting antibacterial activity. To date, MET has been used as a primary antibiotic in the second-line treatment of $\mathrm{H}$. pylori infection. However, MET has a higher possibility of conferring antibiotic resistance than other antibiotics. In Korea, the resistance rates of MET have been reported to be between $35.8 \%$ to $56.3 \%$ since 2010 [14,23-27].

A previous study reported that diabetes mellitus may be a factor in reducing the eradication rate in second-line BQT [28]. Additionally, female sex [29-33] and the presence of a gastroduodenal ulcer [34-36] are associated with the failure of $\mathrm{H}$. pylori eradication therapy. However, these factors were not significantly associated with the treatment failure of $\mathrm{H}$. pylori infection in this study.

According to the studies that analyzed the results of MET-based quadruple therapy for $\mathrm{H}$. pylori infection, the eradication rates ranged from $74 \%$ to $93.6 \%$ when this regimen was prescribed as a second-line treatment to patients for whom CAM-based triple eradication therapy failed [14,37-39]. These values are consistent with the results of the current study. Although this study analyzed data of patients who underwent 7-day METbased second-line BQT, recent guidelines on $H$. pylori eradication therapy recommend 10- or 14-day, rather than 7-day, eradication for $H$. pylori. Several studies have showed results consistent with these guidelines [27,40].

The results of this study show the importance of determining the history of MET use from patient medical records. Throughout the previous studies, no clinical factor has predicted the failure of MET-based antibacterial treatment as much as the history of MET use. Moreover, MET resistance cannot be predicted by clinically available laboratory tests. For CAM, genetic tests that can determine antibiotic resistance by evaluating a point mutation are available commercially and used in clinical practice [14].

Understanding past antibiotic consumption may be helpful for predicting the outcome and improving the success rate of $H$. pylori eradication therapy. Therefore, confirming a patient's past history of antibiotic use before initiating eradication therapy could yield better treatment outcomes than conventional empirical treat- ment, as recommended in the current guidelines. In addition, the practical difficulty of identifying antibiotic resistance through laboratory testing can be avoided. Although patients may have a history of antibiotic use, antibiotic resistance does not always develop. However, increased antibiotic resistance has been reported in patients with a history of antibiotic use. Antibiotic resistance has also been reported in patients with a history of MET use $[41,42]$. As there is no reliable test to check for antibiotic resistance to MET for $\mathrm{H}$. pylori, a history of MET use has to be utilized as a significant clinical factor to set a strategy for $\mathrm{H}$. pylori eradication as stated in the Maastricht V/Florence Consensus Report [15].

This study has several limitations. Because the data were analyzed retrospectively, other clinical factors, such as compliance, drinking, and smoking, could not be analyzed. In this study, the drug compliance of the 7-day MET-based second-line quadruple therapy based on the medical records in this study was estimated to be $90 \%$, meaning that drug compliance is not considered to affect the results of this study. The side effects of eradication drugs were shown to be mild for most patients in the medical records, and the overall rate of side effects was conjectured as approximately $5 \%$ to $10 \%$. In addition, studies conducted by a single institution, without information regarding the diagnosis or treatment by other hospitals, have limited the participants to those at our center. Therefore, the patient group may not represent the community. Additionally, limiting the patients to those who underwent UBT to determine eradication therapy success may have led to selective bias. However, this decision was made to reduce the disturbances caused by differences in the sensitivity and specificity of various tests used to diagnose H. pylori infection. Because an antibiotic resistance test was not performed, the actual prevalence of antibiotic resistance could not be confirmed.

In conclusion, our study showed that previous MET use was significantly associated with the treatment failure of 7-day MET-based second-line quadruple therapy in $\mathrm{H}$. pylori infection. Therefore, we suggest that any history of MET use must be used to establish a treatment plan for patients who need MET-based therapy for $H$. pylori eradication. A large-scale prospective study should be performed to evaluate the history of MET use on $H$. pylori eradication therapy. 


\section{KEY MESSAGE}

1. Previous administration of metronidazole significantly lowered the Helicobacter pylori eradication rate in patients who had metronidazole-based second-line quadruple therapy.

2. This study provides evidence for why information regarding any previous administration of antibiotics should be included in the process of $\mathrm{H}$. pylori eradication strategy.

3. Further studies investigating optimal eradication strategies for $\mathrm{H}$. pylori, based on the clinical database and including the administration of antibiotics, should be prospectively designed.

\section{Conflict of interest}

No potential conflict of interest relevant to this article was reported.

\section{Acknowledgments}

This work was supported by the new faculty research fund of Ajou University School of Medicine.

\section{REFERENCES}

1. Wang F, Meng W, Wang B, Qiao L. Helicobacter pylori-induced gastric inflammation and gastric cancer. Cancer Lett 2014;345:196-202.

2. Bae JM, Kim EH. Helicobacter pylori infection and risk of gastric cancer in Korea: a quantitative systematic review. J Prev Med Public Health 2016;49:197-204.

3. Yeo SH, Yang CH. Peptic ulcer disease associated with Helicobacter pylori infection. Korean J Gastroenterol 2016;67:289-299.

4. Thung I, Aramin H, Vavinskaya V, et al. Review article: the global emergence of Helicobacter pylori antibiotic resistance. Aliment Pharmacol Ther 2016;43:514-533.

5. Flores-Trevino S, Mendoza-Olazaran S, Bocanegra-Ibarias P, Maldonado-Garza HJ, Garza-Gonzalez E. Helicobacter pylori drug resistance: therapy changes and challenges. Expert Rev Gastroenterol Hepatol 2018;12:819-827.

6. Gong EJ, Yun SC, Jung HY, et al. Meta-analysis of first-line triple therapy for helicobacter pylori eradication in Korea: is it time to change? J Korean Med Sci 2014;29:704-713.

7. Kim JM, Kim JS, Jung HC, Kim N, Song IS. Antibiotic resistance of Helicobacter pylori isolated from Korean patients in 2003. Korean J Gastroenterol 2004;44:126-135.

8. Lee JH, Shin JH, Roe IH, et al. Impact of clarithromycin resistance on eradication of Helicobacter pylori in infected adults. Antimicrob Agents Chemother 2005;49:16001603 .

9. Kim SE, Park MI, Park SJ, et al. Trends in Helicobacter pylori eradication rates by first-line triple therapy and related factors in eradication therapy. Korean J Intern Med 2015;30:801-807.

10. Shin WG, Lee SW, Baik GH, et al. Eradication rates of Helicobacter pylori in Korea over the past 10 years and correlation of the amount of antibiotics use: nationwide survey. Helicobacter 2016;21:266-278.

11. Woo HY, Park DI, Park H, et al. Dual-priming oligonucleotide-based multiplex PCR for the detection of Helicobacter pylori and determination of clarithromycin resistance with gastric biopsy specimens. Helicobacter 2009;14:22-28.

12. Lehours P, Siffre E, Megraud F. DPO multiplex PCR as an alternative to culture and susceptibility testing to detect Helicobacter pylori and its resistance to clarithromycin. BMC Gastroenterol 2011;11:112.

13. Lim SG, Park RW, Shin SJ, et al. The relationship between the failure to eradicate Helicobacter pylori and previous antibiotics use. Dig Liver Dis 2016;48:385-390.

14. An B, Moon BS, Kim H, et al. Antibiotic resistance in Helicobacter pylori strains and its effect on $\mathrm{H}$. pylori eradication rates in a single center in Korea. Ann Lab Med 2013;33:415-419.

15. Malfertheiner P, Megraud F, O'Morain CA, et al. Management of Helicobacter pylori infection-the Maastricht V/ Florence Consensus Report. Gut 2017;66:6-30.

16. Calvet X, Garcia N, Lopez T, Gisbert JP, Gene E, Roque M. A meta-analysis of short versus long therapy with a proton pump inhibitor, clarithromycin and either metronidazole or amoxycillin for treating Helicobacter pylori infection. Aliment Pharmacol Ther 2000;14:603-609.

17. Ford A, Moayyedi P. How can the current strategies for Helicobacter pylori eradication therapy be improved? Can J Gastroenterol 2003;17 Suppl B:36B-40B.

18. Flores HB, Salvana A, Ang ELR, et al. M1138 Duration of proton-pump inhibitor-based triple therapy for Helico- 
bacter pylori eradication: a meta-analysis. Gastroenterology 2010;138(5 Suppl 1):S-340.

19. Malfertheiner P, Bazzoli F, Delchier JC, et al. Helicobacter pylori eradication with a capsulecontaining bismuth subcitrate potassium, metronidazole, and tetracycline given with omeprazole versus clarithromycin-based triple therapy: a randomised, open-label, non-inferiority, phase 3 trial. Lancet 2011;377:905-913.

20. Ford AC, Malfertheiner P, Giguere M, Santana J, Khan M, Moayyedi P. Adverse events with bismuth salts for Helicobacter pylori eradication: systematic review and meta-analysis. World J Gastroenterol 2008;14:7361-7370.

21. Fischbach L, Evans EL. Meta-analysis: the effect of antibiotic resistance status on the efficacy of triple and quadruple first-line therapies for Helicobacter pylori. Aliment Pharmacol Ther 2007;26:343-357.

22. Graham DY, Shiotani A. Which therapy for Helicobacter pylori infection? Gastroenterology 2012;143:10-12.

23. Lee JW, Kim N, Kim JM, et al. Prevalence of primary and secondary antimicrobial resistance of Helicobacter pylori in Korea from 2003 through 2012. Helicobacter 2013;18:206-214.

24. Choi WJ, Do GW, Lee GH. Changes in the antibiotic resistance rates of Helicobacter pylori strains isolated in tertiary medical institutions in Seoul. Korean J Med 2014;86:308-313.

25. Chung JW, Jung YK, Kim YJ, et al. Ten-day sequential versus triple therapy for Helicobacter pylori eradication: a prospective, open-label, randomized trial. J Gastroenterol Hepatol 2012;27:1675-1680.

26. Park CS, Lee SM, Park CH, et al. Pretreatment antimicrobial susceptibility-guided vs. clarithromycin-based triple therapy for Helicobacter pylori eradication in a region with high rates of multiple drug resistance. Am J Gastroenterol 2014;109:1595-1602.

27. Lee JY, Kim N, Kim MS, et al. Factors affecting first-line triple therapy of Helicobacter pylori including CY$\mathrm{P}_{2} \mathrm{C} 19$ genotype and antibiotic resistance. Dig Dis Sci 2014;59:1235-1243.

28. Kim SE, Park MI, Park SJ, et al. Second-line bismuth-containing quadruple therapy for Helicobacter pylori eradication and impact of diabetes. World J Gastroenterol 2017;23:1059-1066.

29. Wolle K, Leodolter A, Malfertheiner P, Konig W. Antibiotic susceptibility of Helicobacter pylori in Germany: stable primary resistance from 1995 to 2000. J Med Microbiol
2002;51:705-709.

30. McMahon BJ, Hennessy TW, Bensler JM, et al. The relationship among previous antimicrobial use, antimicrobial resistance, and treatment outcomes for Helicobacter pylori infections. Ann Intern Med 2003;139:463-469.

31. Zullo A, Perna F, Hassan C, et al. Primary antibiotic resistance in Helicobacter pylori strains isolated in northern and central Italy. Aliment Pharmacol Ther 2007;25:14291434 .

32. Parsons HK, Carter MJ, Sanders DS, Winstanley T, Lobo AJ. Helicobacter pylori antimicrobial resistance in the United Kingdom: the effect of age, sex and socio-economic status. Aliment Pharmacol Ther 2001;15:1473-1478.

33. Prazeres Magalhaes P, De Magalhaes Queiroz DM, Campos Barbosa DV, et al. Helicobacter pylori primary resistance to metronidazole and clarithromycin in Brazil. Antimicrob Agents Chemother 2002;46:2021-2023.

34. Megraud F, Coenen S, Versporten A, et al. Helicobacter pylori resistance to antibiotics in Europe and its relationship to antibiotic consumption. Gut 2013;62:34-42.

35. Wong WM, Xiao SD, Hu PJ, et al. Standard treatment for Helicobacter pylori infection is suboptimal in non-ulcer dyspepsia compared with duodenal ulcer in Chinese. Aliment Pharmacol Ther 2005;21:73-81.

36. Chung SJ, Lee DH, Kim N, et al. Eradication rates of helicobacter pylori infection with second-line treatment: non-ulcer dyspepsia compared to peptic ulcer disease. Hepatogastroenterology 2007;54:1293-1296.

37. Hojo M, Miwa H, Nagahara A, Sato N. Pooled analysis on the efficacy of the second-line treatment regimens for Helicobacter pylori infection. Scand J Gastroenterol 2001;36:690-700.

38. Graham DY. Efficient identification and evaluation of effective Helicobacter pylori therapies. Clin Gastroenterol Hepatol 2009;7:145-148.

39. Lee BH, Kim N, Hwang TJ, et al. Bismuth-containing quadruple therapy as second-line treatment for Helicobacter pylori infection: effect of treatment duration and antibiotic resistance on the eradication rate in Korea. Helicobacter 2010;15:38-45.

40. Zullo A, De Francesco V, Bellesia A, et al. Bismuth-based quadruple therapy following $H$. pylori eradication failures: a multicenter study in clinical practice. J Gastrointestin Liver Dis 2017;26:225-229.

41. Perez Aldana L, Kato M, Nakagawa S, et al. The relationship between consumption of antimicrobial agents and 
the prevalence of primary Helicobacter pylori resistance. Helicobacter 2002;7:306-309.

42. Ortiz V, Estevez-Ordonez D, Montalvan-Sanchez E, et al.
Helicobacter pylori antimicrobial resistance and antibiotic consumption in the low-resource Central America setting. Helicobacter 2019;24:e12595. 